\title{
The Aerogel RICH detector of the Belle II experiment
}

\section{Luka Šantelj ${ }^{a b *}$}

Jozef Stefan Institute and University of Ljubljana

E-mail: luka.santeljeijs.si

I. Adachic ${ }^{i}$, L. Burmistrov ${ }^{d}$, F. Le Diberder ${ }^{d}$, R. Dolenec ${ }^{b}$, K. Hataya ${ }^{e}$, T. lijima $^{f}$, S. Kakimoto ${ }^{e}$, H. Kakuno ${ }^{e}$, H. Kawai ${ }^{g}$, T. Kawasaki ${ }^{h}$, H. Kindo ${ }^{i}$, T. Kohriki ${ }^{c}$, T. Konno ${ }^{h}$, S. Korpar ${ }^{a j}$, E. Kou ${ }^{d}$, P. Križan ${ }^{a b}$, T. Kumita ${ }^{e}$, Y. Lai ${ }^{c}$, M. Machida ${ }^{k}$, M. Mrvar ${ }^{a}$, S. Nishida ${ }^{c i}$, K. Noguchi ${ }^{e}$, K. Ogawa $^{l}$, S. Ogawa ${ }^{m}$, R. Pestotnik ${ }^{a}$, M. Shoji ${ }^{c}$, T. Sumiyoshi' ${ }^{e}$, M. Tabata ${ }^{g}$, S. Tamechika ${ }^{e}$, M. Yonenaga $^{e}$, M. Yoshizawa ${ }^{l}$, Y. Yusa ${ }^{l}$

a Jožef Stefan Institute, Ljubljana, Slovenia

${ }^{b}$ University of Ljubljana, Slovenia

${ }^{c}$ High Energy Accelerator Research Organization (KEK), Tsukuba, Japan

${ }^{d}$ Laboratoire de Laccelerateur Lineaire (LAL), Orsay, France

e Tokyo Metropolitan University, Hachioji, Japan

${ }^{f}$ Nagoya University, Nagoya, Japan

${ }^{g}$ Chiba University, Chiba, Japan

${ }^{h}$ Kitasato University, Sagamihara, Japan

${ }^{i}$ SOKENDAI (The Graduate University of Advanced Studies), Tsukuba, Japan

j University of Maribor, Slovenia

${ }^{k}$ Tokyo University of Science, Noda, Japan

${ }^{l}$ Niigata University, Niigata, Japan

${ }^{m}$ Toho University, Funabashi, Japan

In the forward end-cap of the Belle II spectrometer, an innovative proximity focusing Ring Imaging Cherenkov counter with a multilayer focusing aerogel radiator has been installed. The detector is designed to be operated in a magentic field of $1.5 \mathrm{~T}$, and consists of a double layer aerogel radiator, an expansion volume and a photon detector. In total 420 Hamamatsu hybrid avalanche photo sensors with 144 channels each are used to read out single Cherenkov photons with high efficiency. We expect the device to provide better than $4 \sigma$ separation of pions from kaons in the full kinmatic region of the experiment, from $0.5 \mathrm{GeV} / c$ to $4 \mathrm{GeV} / c$. The detector components have been successfully produced and installed in the spectrometer. After the commissioning phase in 2018, the detector is now included in the Belle II data taking, and is expected to contribute substantially to the performance of the spectrometer in looking for rare decays of $B$ and $D$ mesons, and of tau leptons. In this contribution we review the detector design and present results of the first detector performance studies.

European Physical Society Conference on High Energy Physics - EPS-HEP2019 -

10-17 July, 2019

Ghent, Belgium

* Speaker. 


\section{Introduction}

The Belle II experiment is a next generation $B$ physics experiment, operating at the SuperKEKB asymmetric energy $e^{+} e^{-}$collider (Super $B$-factory) at KEK, Japan [1]. It started its operation in 2019 with the goal of collecting $50 \mathrm{ab}^{-1}$ of data by the 2027 (50x Belle's data sample). This unprecedentedly large collection of decays of $B, D$ mesons and $\tau$ leptons will allow for high precision tests of the flavor sector of the Standard Model, potentially leading to the discovery of new physics phenomena.

One of the critical aspects at Belle II, in order to exploit its full physics potential, is efficiency of its particle identification (PID) systems. Particle identification, mostly discrimination between pions and kaons, is crucial not only for reducing background levels, but also for the neutral $B$ meson flavor tagging (to discriminate $B^{0}$ and $\bar{B}^{0}$ ) essential to many CP-violation related measurements. In addition, excellent lepton identification is required for measurements of possible lepton flavor violating decays and tests of lepton flavor universality. At Belle II PID is provided by the time-ofpropagation counter (TOP) in the barrel and aerogel RICH counter (ARICH) in the end-cap region [2]. The goal of the ARICH is mainly to provide very good $\pi / K$ separation in the full kinematic range of the experiment $(4 \sigma$ separation at momenta between 0.5 and $3.5 \mathrm{GeV} / c)$, and to provide discrimination between $\pi, \mu$ and $e$ below $1 \mathrm{GeV} / c$ (relevant for rare decays, such as $B \rightarrow K l l$ ).

In the following sections we describe the ARICH design along with operational experience from the first period of Belle II data taking (March-July 2019). We conclude with results of the first detector performance studies.

\section{Detector design and components}

The ARICH is a proximity focusing RICH with aerogel as a radiator. Its design and selection of components were mainly guided by the limited available space, operation in the magnetic field of $1.5 \mathrm{~T}$, and radiation hardness (to withstand neutron fluence of $>10^{11} 1 \mathrm{MeV} n \mathrm{eq} / \mathrm{cm}^{2} /$ year). The main detector components and their geometrical configuration are shown on the left picture of figure 1 .

The radiator plane is covered with two layers of wedge shaped aerogel tiles (in total $2 \times 124$ tiles are used, each of approximate size of $18 \times 18 \times 2 \mathrm{~cm}$ ), with refractive indices of $n_{1}=1.045$ in the first and $n_{2}=1.055$ in the second layer. These refractive indices are chosen in a way that the Cherenkov rings from the first and second layer overlap on the detector plane, which significantly improves the Cherenkov angle resolution [3]. The middle picture of figure 1 shows the radiator plane just before it was joint with the photon detector plane, shown on the right picture. The photon detector plane is covered with 420 HAPDs (Hybrid Avalanche Photo Detector [4, 5]), arranged in 7 concentric rings. The HAPD consists of a vacuum tube (size of $7.3 \times 7.3 \times 2.0 \mathrm{~cm}$ ) with bi-alkali photo-cathode on the bottom side of quartz window and pixelated APD with 144 channels $(\sim 5 \times 5$ $\mathrm{mm}$ ) at the bottom of the tube. The total gain of about $6 \times 10^{4}$ is provided by the high voltage of 8 $\mathrm{kV}$ applied to the tube and bias voltage of $\sim 300 \mathrm{~V}$ applied to the APD. The quantum efficiency of photo-cathode is $\sim 30 \%$ at $400 \mathrm{~nm}$, and the active area is about $65 \%$ of package size. The description of the photon detectors readout system can be found elsewhere [6]. On the outer side 
of the ARICH 18 planar mirrors are mounted to reflect the photons that would otherwise be lost back to the photo-sensitive area.

During the first period of Belle II data taking we did not experience any major problem in the detector operation. The fraction of non-functional HAPD channels was about $1 \%$, mostly on the account of broken APD chips (to which bias voltage can not be applied) or individual broken channels in the front-end readout ASICs.
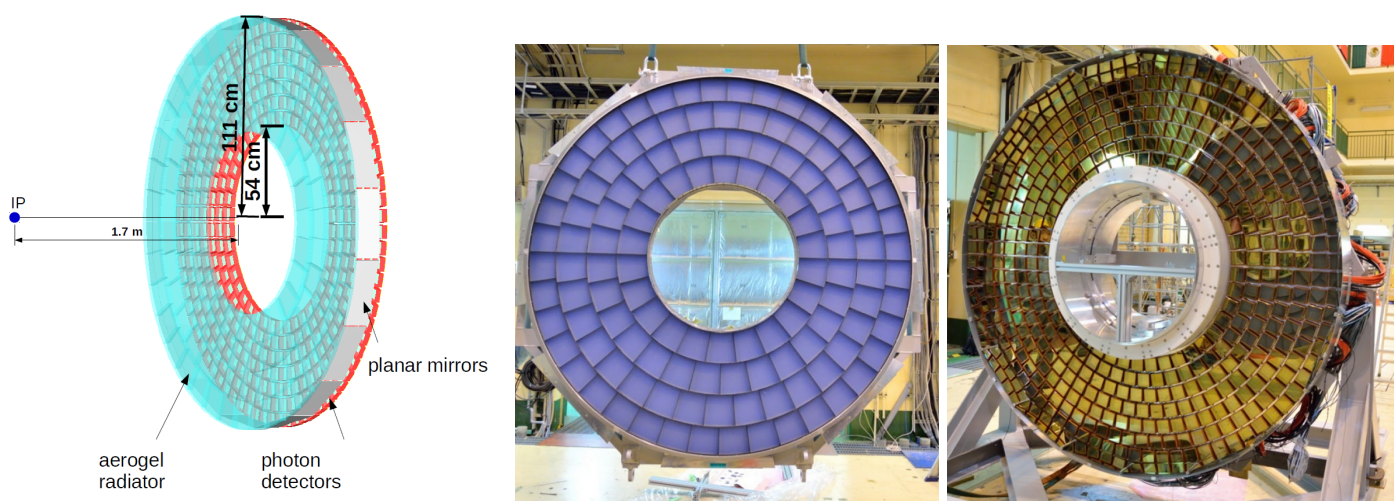

Figure 1: Left: Sketch of geometry configuration of ARICH with its main components. Middle: Radiator plane covered with two layers of aerogel tiles. Right: Photon detector plane covered with 420 HAPDs.

\section{Detector performance}

In this section we describe results of the first detector performance studies. In the first part we compare the Cherenkov rings generated by high momentum muons as seen in the measured data and in the detector simulation. In the second part we study the detector PID performance using independently tagged kaon and pion tracks. The results are based on the data sample corresponding to the integrated luminosity of $1.3 \mathrm{fb}^{-1}$ (while in total about $6.5 \mathrm{fb}^{-1}$ was collected in the 2019 spring run).

\subsection{Cherenkov angle distribution in $e^{+} e^{-} \rightarrow \mu^{+} \mu^{-}$events}

Using large number of available muon tracks originating from the $e^{+} e^{-} \rightarrow \mu^{+} \mu^{-}$events we performed a detailed study comparing the Cherenkov ring image in measured and simulated events. Muons from these events are nearly monochromatic with the energy of about $7 \mathrm{GeV}$, producing essentially saturated Cherenkov rings in the ARICH. The left two plots of figure 2 show obtained Cherenkov ring image in the angular coordinate system of a track ${ }^{1}$ for the measured and simulated data. The image is obtained as a normalized sum of Cherenkov rings from $\mathscr{O}(10 \mathrm{k})$ muon tracks. Several detailed features, such as Cherenkov photons produced in the quartz window of photon detectors and "echo ring" originating from the non-converted photons reflected from the APD surface back to the photo-cathode, can be seen to be well reproduced in the simulation. The right plot of figure 2 shows the rings integrated over the azimuthal angle. We determine the number of

\footnotetext{
${ }^{1}$ In this system the track direction corresponds to $(0,0)$ and the distance from the origin shows the photon direction polar angle, i.e. Cherenkov angle.
} 
signal Cherenkov photons and the Cherenkov angle resolution for measured data and simulation by fitting the obtained distributions with a single Gaussian function for the peak and a first order polynomial for the background distribution. The obtained numbers of signal photons per muon track are $N_{\text {sig }}^{\text {data }}=11.38$ and $N_{\text {sig }}^{M C}=11.27$ for the measured and simulated data respectively, while the corresponding Cherenkov angle resolutions (i.e. signal peak width) are $\sigma_{c}^{\text {data }}=12.70 \mathrm{mrad}$ and $\sigma_{c}^{M C}=12.75 \mathrm{mrad}$ (with negligible uncertainties from the fit). Some discrepancy between the measured and simulated data can be seen in the peak produced by charged tracks in the window of photon detectors. This arises due to difficulty of correctly modeling the optical properties of photo-cathode and the response of avalanche photo-diode to large number of photons. Based on these observations mentioned effects will be adjusted for in the detector simulation.
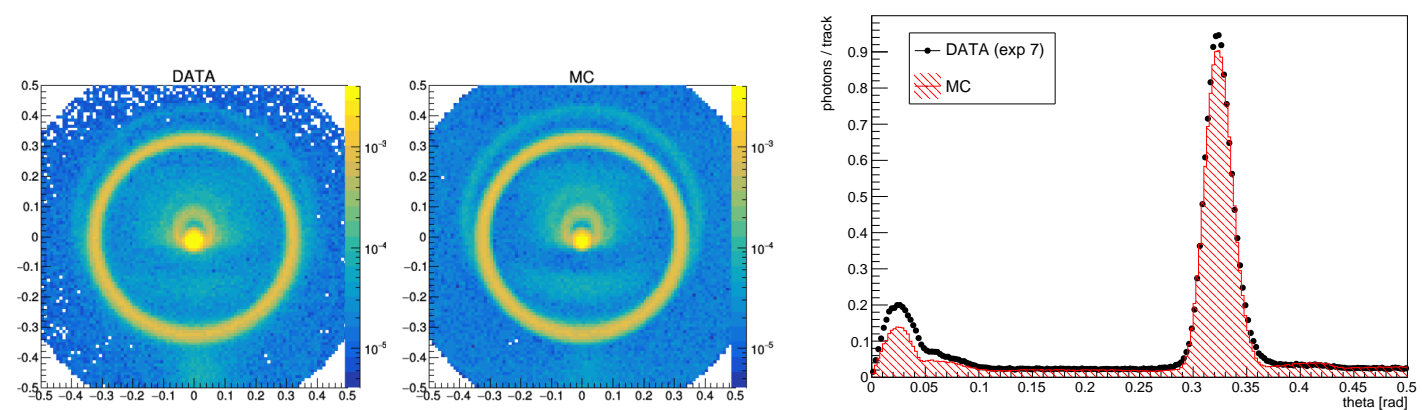

Figure 2: Left: Comparison of Cherenkov ring image as observed in measured data and in detector simulation. Right: Comparison of measured and simulated Cherenkov angle distribution (integral of left plots over the ring azimuthal angle).

\subsection{Particle identification performance}

We estimate the detector ability to discriminate between pions and kaons using pion and kaon tracks from $D^{*+} \rightarrow D^{0}\left[\rightarrow K^{-} \pi^{+}\right] \pi_{\text {slow }}^{+}$and $D^{*-} \rightarrow \bar{D}^{0}\left[\rightarrow K^{+} \pi^{-}\right] \pi_{\text {slow }}^{-}$decay chains. Here, pion and kaon tracks can be identified independently of ARICH information, based on their charge in association with the charge of low momentum pion $\left(\pi_{\text {slow }}^{ \pm}\right)$from $D^{* \pm}$. In addition, the background level in this decay mode can be effectively reduced by requiring the difference of reconstructed invariant masses of $D^{*}$ and $D^{0}$ mesons to be within a narrow window around the expected value $\left(\left|M_{D^{*}}-M_{D^{0}}-145.43 \mathrm{MeV} / c^{2}\right|<1.5 \mathrm{MeV} / c^{2}\right.$ is used). We determine the efficiency of kaon identification $^{2}$ and pion misidentification rate ${ }^{3}$ from the signal yield of reconstructed $D^{0}$ 's, where pion or kaon track used in the reconstruction is required to satisfy the imposed selection criteria on PID likelihood ratio obtained from the ARICH $\left(R[K / \pi]=\mathscr{L}_{K} /\left(\mathscr{L}_{K}+\mathscr{L}_{\pi}\right)\right)$. The $D^{0}$ signal yield is determined from the fit of $D^{0}$ invariant mass distribution using a Gaussian function for the signal and a constant value for the background distributions. An example of such fit is shown in top two plots of figure 3. Left (right) plot shows data distribution and fit of $D^{0}$ invariant mass for candidates

\footnotetext{
${ }^{2} \varepsilon_{K}=N_{K}^{\text {iden. }} / N_{K}^{\text {all }}$, where $N_{K}^{\text {iden. }}$ is the number of correctly identified kaons by ARICH and $N_{K}^{\text {all }}$ is the total number of kaons entering ARICH.

${ }^{3} \varepsilon_{\pi}=N_{\pi}^{\text {misiden. }} / N_{\pi}^{\text {all }}$, where $N_{\pi}^{\text {misiden. }}$ is the number of pions identified as kaon by ARICH and $N_{\pi}^{\text {all }}$ is the total number of pions entering ARICH.
} 
with pion (kaon) track entering ARICH. Fainted points and lines show the case when no selection is imposed on $R[K / \pi]$ and solid points and lines after imposing $R[K / \pi]>0.6$. By repeating such fit using different $R[K / \pi]$ criteria we obtain the bottom left plot of figure 3, which shows obtained kaon identification efficiency at different pion misidentification rates. The right bottom plot shows dependency of these two quantities on the track momentum, where fixed criteria of $R[K / \pi]>0.6$ is used at all points. The obtained performance is slightly lower than expected from the simulations (up to few $\%$ in $\mathrm{K}$ id. efficiency), mostly on the account of only very preliminary detector calibrations available at the time of this study.
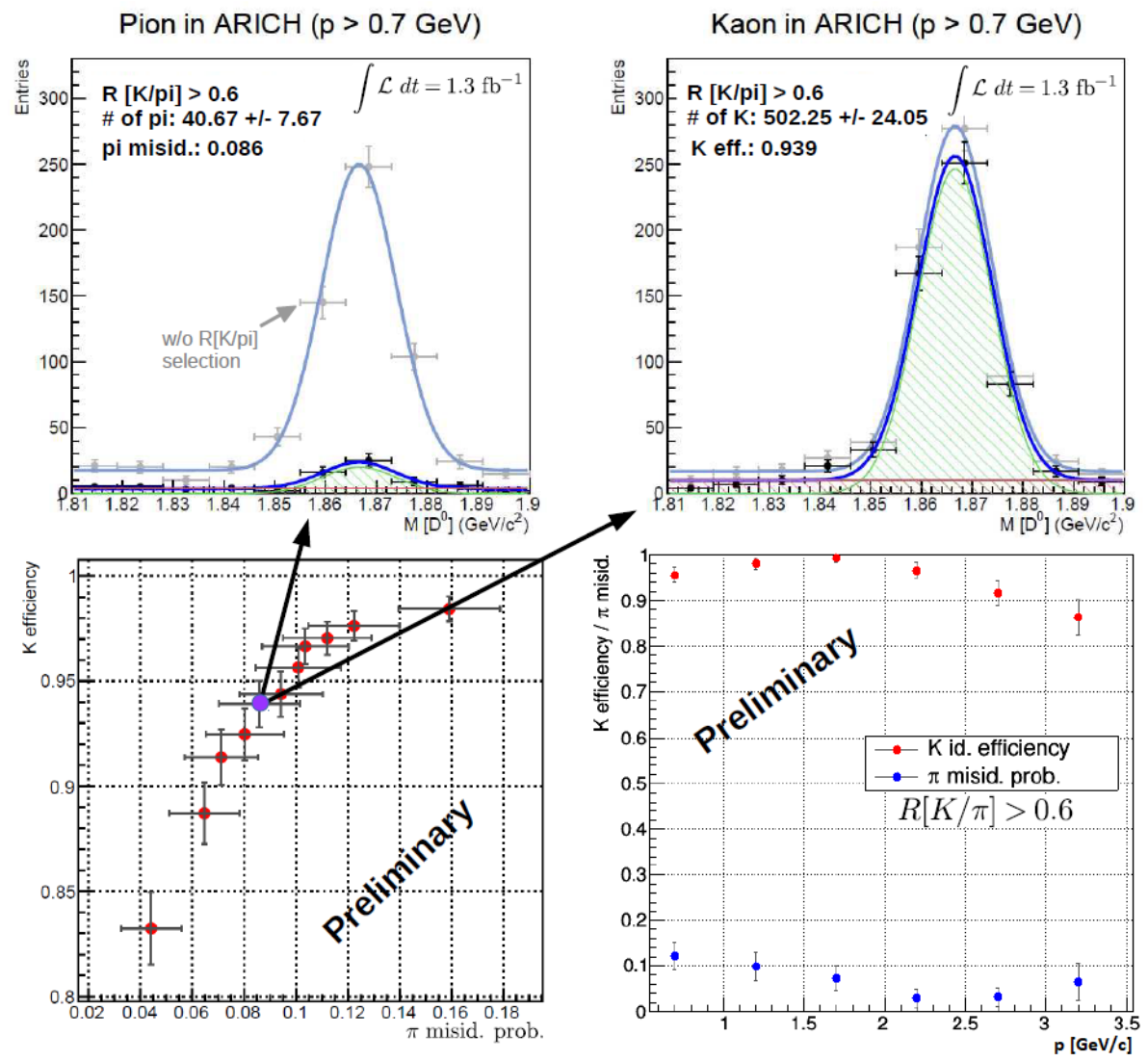

Figure 3: Upper: Example of fits of $D^{0}$ invariant mass distribution which allow us to determine kaon identification efficiency and pion misidentification rate at given criteria on $R[K / \pi]$. Bottom left: Kaon identification efficiency versus pion misidentification rate for all kaon/pion tracks from $D^{0}$ decays that enter ARICH. Bottom right: Kaon identification efficiency and pion misidentification rate as a function of track momentum.

\section{Summary}

Aerogel RICH detector was installed in the forward end-cap of the Belle II spectrometer, with the main goal of providing excellent discrimination between pions and kaons in the full kinematic range of the experiment (up to $4 \mathrm{GeV} / \mathrm{c}$ ). During the early period of Belle II data taking the detector performed well, without significant problems. With only preliminary detector calibrations available 
the performance is already relatively close to expected from the simulations. In the future our focus will be on improving calibration methods and data reconstruction algorithms to provide even better PID performance of the ARICH detector.

\section{References}

[1] BELLE-II collaboration, Belle II Technical Design Report, 1011.0352.

[2] S. Nishida et al., Aerogel RICH for the Belle II forward PID, Nucl. Instrum. Meth. A766 (2014) 28 .

[3] S. Korpar et al., Novel type of proximity focusing RICH counter with multiple refractive index aerogel radiator, Nucl. Instrum. Meth. A572 (2007) 429.

[4] S. Korpar et al., A 144-channel HAPD for the Aerogel RICH at Belle II, Nucl. Instrum. Meth. A766 (2014) 145.

[5] I. Adachi et al., Study of 144-channel multi-anode hybrid avalanche photo-detector for the Belle RICH counter, Nucl. Instrum. Meth. A623 (2010) 285.

[6] R. Pestotnik et al., Front-end electronics of the belle ii aerogel ring imaging detector, Nuclear Instruments and Methods in Physics Research Section A: Accelerators, Spectrometers, Detectors and Associated Equipment (2018) . 